\title{
Efeito da Condição Corporal ao Parto sobre Alguns Parâmetros do Metabolismo Energético, Produção de Leite e Incidência de Doenças no Pós-Parto de Vacas Leiteiras
}

\author{
Ernani Paulino do Lago1, Alexandre Vaz Pires², Ivanete Susin², Vidal Pedroso de Faria², \\ Luiz Alberto do Lago ${ }^{3}$
}

\begin{abstract}
RESUMO - Cento e dezoito vacas da raça Holandesa foram utilizadas para se avaliar o efeito da condição corporal ao parto sobre a produção e teor de gordura no leite, níveis plasmáticos de beta-hidroxibutirato (BHBA), glicose e aspartato-aminotransferase (AST) e incidência de doenças no início da lactação. De acordo com seu escore de condição corporal (ECC) ao parto, em uma escala de 1 (magra), a 5 (gorda), as vacas foram divididas em dois grupos: I) constituído por vacas com ECC $\geq 4,0$ ); II) vacas com ECC $\leq 3,5$. Os grupos foram diferentes entre si e apresentaram ECC médio de 4,2 e 3,2 para os grupos I e II, respectivamente. O ECC ao parto não afetou a produção de leite, gordura do leite, glicose e AST plasmáticas e a incidência de doenças no período pós-parto. Entretanto, o BHBA foi mais alto $(\mathrm{P}<0,0084)$ e a perda de peso foi mais intensa $(\mathrm{P}<0,01)$ no grupo I. Quando analisada cada semana separadamente, o BHBA do grupo I foi maior durante as semanas $1(\mathrm{P}<0,009), 2(\mathrm{P}<0)$ e $3(\mathrm{P}<0,004)$ pós-parto, sendo que o pico ocorreu na primeira e segunda semana para ambos os grupos. O grupo I também apresentou teores de glicose significativamente menores durante a primeira e segunda semanas $(\mathrm{P}<0$ e $\mathrm{P}<0,008$, respectivamente) e numericamente mais baixos nas demais semanas, demonstrando um balanço energético negativo mais intenso neste período.
\end{abstract}

Palavras-chave: condição corporal, vacas leiteiras

\section{Effect of Body Condition Score at Calving on Energy Metabolism, Milk Yield and Disease Occurence in Postpartum of Dairy Cows}

\begin{abstract}
One hundred and eighteen Holstein cows were used to evaluate the effects of body condition score at parturition on milk production and fat content, blood beta-hydroxybutirate (BHBA), glucose and aspartate-aminotransferase (AST) and disease occurence in early lactation. According to their body condition score (BCS) at calving, 1 (thin) at 5 (fat), the cows were alloted in two groups: I) composed by BCS $\geq 4,0$; and II) composed by BCS $\leq 3,5$. The BCS were 4.2 and 3.2 for the group I and II, respectively. The BCS at calving did not affect milk production, milk fat, plasma glucose and AST and disease occurence in early lactation. However, the group I had higher $(\mathrm{P}<0.0084)$ BHBA and lost more $(\mathrm{P}=0.01)$ weight. When the analysis was performed weekly the BHBA for the group I was higher until three weeks post-partum, showing a peak at first and second week for both groups. The group I also had lower levels of plasma glucose in the first and second weeks, showing a intense negative energy balance in this period.
\end{abstract}

Key Words: body condition score, dairy cows

\section{Introdução}

Nos últimos anos tem sido estudada a relação do escore de condição corporal (ECC) ao parto com o desempenho da lactação e o aparecimento de doenças no pós-parto. O ECC é uma maneira subjetiva de se avaliar as reservas energéticas da vaca, sendo baseado na observação visual e palpação de áreas específicas para avaliar os depósitos de tecido adiposo e massa muscular.

O ECC varia de acordo com o método adotado, porém valores mais altos sempre indicam vacas com mais reserva corporal. WILDMAN et al. (1982) e
EDMONSON et al. (1989) propuseram uma escala de 1 a 5 com intervalo de 0,25 pontos para medir o ECC, sendo este um método bastante utilizado nos Estados Unidos.

PATTON et al. (1988) sugeriram que as vacas ao parirem sem adequada reserva corporal podem ser mais propensas às doenças infecciosas, transtornos metabólicos, baixa eficiência reprodutiva e redução na produção de leite, enquanto vacas excessivamente gordas estariam mais predispostas a dificuldades de parto, síndrome da vaca gorda e, às vezes, morte. EDMONSON et al. (1989) verificaram que as vacas com condição corporal elevada $(4-4,25)$ são mais propensas à cetose, devido ao fato de terem menor

\footnotetext{
1 Universidade Federal de Viçosa - Departamento de Veterinária - CEP 36571-000 Viçosa - MG. E.mail: eplago@mail.ufv.br 2 Departamento de Produção Animal - Escola Superior de Agricultura Luiz de Queiroz - ESALQ - USP - Piracicaba - SP.

3 Departamento de Veterinária - Universidade Federal de Minas Gerais - UFMG - Belo Horizonte - MG.
} 
consumo logo após o parto e mobilizam mais reservas corporais.

Segundo FERGUSON et al. (1994), tanto as vacas muito gordas como muito magras correm o risco de ter problemas metabólicos e doenças, redução na produção de leite e na taxa de concepção e dificuldade em parir. Estes mesmos autores sugeriram os seguintes valores para o ECC de novilhas e de vacas nos seus diferentes estádios de produção.

\begin{tabular}{lcc} 
Estádio da lactação & ECCideal & Intervalo sugerido \\
\hline Período seco & 3,50 & $3,25-3,75$ \\
Parto & 3,50 & $3,25-3,75$ \\
Início da lactação & 3,00 & $2,50-3,25$ \\
Meio da lactação & 3,25 & $2,75-3,25$ \\
Fim da lactação & 3,50 & $3,00-3,50$ \\
Novilhas em crescimento & 3,00 & $2,75-3,25$ \\
Novilhas ao parto & 3,50 & $3,25-3,75$ \\
\hline
\end{tabular}

DOMECQ et al. (1997), estudando a relação entre ECC e gordura subcutânea em vacas, determinada pela técnica de ultrassonografia, concluíram que o ECC refletiu adequadamente as reservas de gordura neste tecido.

As mudanças no ECC entre o final da gestação, parto e início da lactação têm sido responsabilizadas por baixar o desempenho produtivo e reprodutivo pós-parto e aumentar a incidência de transtornos metabólicos em vacas de leite (WILDMAN et al., 1982; RUEGG e MILTON, 1995). Vacas que pariram com ECC próximo de 4,0 foram consideradas propensas a terem mais problemas de mastite, doenças metabólicas e problemas reprodutivos (CURTIS et al., 1985; PATTON et al., 1988; FERGUSON et al., 1994). Todavia, GARNSWORTHY e TOPPS (1987) não puderam associar alta condição corporal ao parto e incidência de doenças metabólicas. Adicionalmente, BUTLER E SMITH (1989) ao estudarem o efeito de diferentes ECC ao parto sobre o desempenho pós-parto, não observaram diferença com relação à incidência de transtornos no metabolismo.

RUEGG e MILTON (1995) utilizaram 429 vacas para determinar a relação da perda de ECC com produção de leite, performance reprodutiva e incidência de doenças. Os autores concluíram não ter havido diferença na perda de condição corporal entre os animais que tiveram doença metabólica e animais sadios, e que o ECC ao parto não influenciou a performance da lactação. Isto concorda com os resultados apresentados por GEARHART et al. (1990), que também relataram não haver diferença entre o ECC ao parto e o risco de doenças metabólicas.

SANTOS (1996), avaliando o efeito de ECC ao parto sobre o desempenho da lactação, não observou diferença na produção ou composição do leite entre dois grupos de vacas consideradas gordas e magras. Também, a incidência de doenças metabólicas, problemas reprodutivos e desempenho reprodutivo não foram afetados. Entretanto, observou-se que as vacas mais gordas perderam mais ECC e tinham níveis de beta-hidroxibutirato (BHBA) mais altos.

Os diferentes resultados com trabalhos envolvendo ECC podem ser devidos aos diferentes métodos adotados para avaliação do ECC e às diferenças de manejo, alimentação e produção entre rebanhos estudados. De certa forma, a avaliação do ECC no pósparto pode ser útil como estratégia para se evitar que as vacas venham a parir muito gordas ou muito magras (FERGUSON, 1991).

O objetivo deste trabalho foi estudar o efeito da condição corporal ao parto sobre alguns parâmetros do metabolismo energético, produção de leite e incidência de doenças no início da lactação.

\section{Material e Métodos}

Cento e dezoito vacas da raça Holandesa, que pariram entre cinco de fevereiro a 24 de junho de 1996, foram estudadas com relação ao ECC, produção de leite, teor de gordura no leite, perda de peso e condição corporal, níveis plasmáticos de BHBA, glicose e aspartato-aminotransferase (AST), e incidência de doenças durante as oito primeiras semanas de lactação.

Os animais pertenciam a um rebanho comercial situado a noroeste do estado de São Paulo. Durante o período experimental a produção média diária de leite foi de $8011 \mathrm{~kg}$, tendo em média 350 vacas em lactação (média de $22,9 \mathrm{~kg} / \mathrm{vaca} / \mathrm{dia}$ ).

As instalações eram do tipo "free-stall" e os animais permaneciam agrupados em lotes de acordo com a produção de leite. As vacas foram ordenhadas três vezes ao dia (4, 12 e 20 horas) e receberam alimentação completa com três refeições diárias (4, 10 e 16 horas), em quantidade suficiente para exceder em torno de $5 \%$ do consumo voluntário, atendendo ou excedendo às exigências para vacas em lactação (NATIONAL RESEARCH COUNCIL - NRC, 1989). 
Para se avaliar o efeito da condição corporal ao parto sobre a produção de leite e teor de gordura no leite, níveis plasmáticos de BHBA, glicose e AST e incidência de doenças, as vacas foram divididas em dois grupos, de acordo com sua condição corporal no dia do parto: I) vacas com $E C C \geq 4,0$; II) vacas com $\mathrm{ECC} \leq 3,5$. Os tratamentos foram diferentes e apresentaram valores médios de 4,2 e 3,2 para os grupos I e II, respectivamente.

Durante o período experimental, do parto à $8^{\mathrm{a}}$ semana de lactação, as vacas foram submetidas semanalmente às seguintes avaliações e colheita de amostras:

a) pesagem individual da produção de leite;

b) avaliação da condição corporal, feita por um único avaliador, utilizando uma escala de 1 (magra) a 5 (gorda), com intervalo de 0,25 pontos, de acordo com o critério proposto por FERGUSON et al. (1994);

c) colheita de amostra de sangue, à vácuo, em recipiente contendo antiglicolítico (fluoreto de sódio) e anticoagulante (heparina), para determinação de BHBA, glicose e AST. Estas amostras foram colhidas sempre no mesmo horário (de 9 às 10 horas da manhã), em relação ao manejo e alimentação dos animais na tentativa de minimizar o efeito das variações diurnas que ocorrem nos níveis plasmáticos destas substâncias por influência do estado de jejum ou pós-prandial. As amostras foram mantidas em gelo até o término da colheita (menos de1 hora) e após centrifugadas a 1100 x g. O plasma obtido foi dividido em alíquotas e armazenadas a $-20^{\circ} \mathrm{C}$ em tubos criogênicos de $1,5 \mathrm{ml}$ para posterior análise;

d) colheita de amostra de leite para determinação de gordura, e determinação semiquantitativa de acetona e acetoacetato através dos testes de campo;

e) avaliação do estado geral de saúde utilizando os métodos semiológicos rotineiros, dando atenção especial às enfermidades de maior ocorrência neste período como distocia, retenção das membranas fetais, infecção uterina, paresia da parturiente, problemas de pé ou casco, mastite e cistos ovarianos.

As vacas foram pesadas logo após o parto, na quarta e na oitava semana de lactação para verificação da variação de peso.

Amostras do alimento foram colhidas semanalmente e armazenadas a $-20^{\circ} \mathrm{C}$ para posterior análise bromatológica. As amostras de ração completa fo- ram compostas em 10 amostras, representando cada duas semanas consecutivas do experimento e analisadas para se obter matéria seca (MS), proteína bruta, extrato etéreo, extrativo não nitrogenado e cinzas segundo a AOAC (1990). Fibra em detergente ácido e fibra em detergente neutro foram determinadas pelo método de VAN SOEST et al. (1991). Após o término do período experimental, as amostras de plasma, leite e alimento foram submetidas às análises de laboratório. A porcentagem de gordura do leite foi obtida utilizando-se o método de separação por ácido sulfúrico.

Os níveis plasmáticos de BHBA foram obtidos utilizando-se o Kit n ${ }^{\circ}$ 310-A (Sigma Diagnostics, P.O. BOX 14508, St. Louis, MO 63178, USA), que se baseia no método enzimático descrito por WILLIANSON et al. (1962). Os resultados foram dados em miligramas por decilitro (mg/dL).

A glicose foi quantificada enzimaticamente utilizando o Kit No 315-100 (Sigma Diagnostics, P.O BOX 14508, St. Louis, MO 63178, USA), que segue o método da glicose oxidase descrito por TIETZ (1982), adaptado para ser usado em placas de microtítulo e posterior leitura em aparelho do tipo "Elisa Reader", que apresenta os resultados em mg/dL.

A enzima hepática aspartato-aminotransferase foi determinada utilizando o Kit AST Reagent - IFCC (Ciba Corning Diagnóstics Corp., Oberlin, Ohio, 44074, USA), que apresenta os resultados em Unidades Internacionais (UI).

Os dados obtidos foram analisados de acordo com o esquema "Split-plot", no qual as vacas foram divididas em dois grupos, de acordo com a condição corporal ao parto (I e II), efeito este considerado como parcela ou "Whole-plot", e as medidas repetidas na mesma vaca (semanas) como subparcelas.

A análise foi feita usando o PROC MIXED (LATOUR, 1994), considerando efeitos fixos de grupo, semana e interação grupo x semana.

Os efeitos de vacas dentro dos grupos foram considerados como aleatórios.

As médias ajustadas (LSMeans) dos efeitos fixos foram comparadas por intermédio da diferença mínima significativa (LSD), obtida pelo teste " $t$ ".

A incidência de doenças nos grupos foi comparada pelo teste de Qui-Quadrado $\left(\chi^{2}\right)$.

\section{Resultados e Discussão}

A composição da dieta está apresentada na Tabela 1. Analisando a média geral das 8 semanas (Tabela 2), 
Tabela 1 - Composição da dieta

Table 1 - Diet composition

\begin{tabular}{|c|c|}
\hline $\begin{array}{l}\text { Ingredientes } \\
\text { Ingredients }\end{array}$ & $\begin{array}{c}\% \text { da } \mathrm{MS} \\
D M \%\end{array}$ \\
\hline Silagem de milho ${ }^{1}$ & 44,70 \\
\hline Corn silage & \\
\hline Milho moído & 28,28 \\
\hline Ground corn & \\
\hline Soja grão & 7,62 \\
\hline Whole soybean & \\
\hline Farelo de soja ${ }^{2}$ & 9,52 \\
\hline Soybean meal & \\
\hline Caroço de algodão & 7,62 \\
\hline Cottonseed & \\
\hline Minerais e vitaminas ${ }^{3}$ & 2,24 \\
\hline Minerals \& Vitamins & \\
\hline Composição química & \\
\hline Chemical composition & \\
\hline Matéria seca & 48,0 \\
\hline Dry matter & \\
\hline Proteína bruta & 16,0 \\
\hline Crude protein & \\
\hline $\mathrm{FDN}^{4}$ & 38,89 \\
\hline$N D F$ & \\
\hline $\mathrm{FDA}^{5}$ & 23,47 \\
\hline$A D F$ & \\
\hline Extrato etéreo & 4,5 \\
\hline Ether extract & \\
\hline $\begin{array}{l}\text { Matéria mineral } \\
\text { Ash }\end{array}$ & 5,5 \\
\hline Extrativo não-nitrogenado & 53,0 \\
\hline Nitrogen free extract & \\
\hline $\mathrm{NDT}^{6}$ & 69,0 \\
\hline$T D N$ & \\
\hline
\end{tabular}

1 Silagem de milho contendo uréia (Corn silage with $5 \%$ urea on fed basis).

${ }^{2}$ Farelo de soja com $45 \%$ de proteína bruta (Soybean meal with $45 \%$ crude protein).

${ }^{3}$ Fósforo, 5.5\%; Cálcio, 22\%; Magnésio, 3.5\%; Enxofre, 2.2\%; Sódio, 7.0\%; Cloreto, 10.8\%; Manganês, 1250 ppm; Ferro, 500 ppm; Cobre, 450 ppm; Zinco, 1550 ppm; Selênio, 20 ppm; Vit. A, 90000 $\mathrm{Ul} / \mathrm{kg}$; Vit. D, $75000 \mathrm{Ul} / \mathrm{kg}$,; Vit. E $1000 \mathrm{Ul} / \mathrm{kg}$.

${ }^{4}$ Fibra em detergente neutro (Neutral detergent fiber).

${ }^{5}$ Fibra em detergente ácido (Acid detergent fiber).

${ }^{6}$ Nutrientes digestíveis totais -valor estimado (Total digestible nutrientsestimated value).

a produção de leite, o teor de gordura no leite, glicose e AST plasmáticas não foram afetados pelo ECC ao parto.

SANTOS (1996) também não encontrou efeito de ECC ao parto, na produção e composição do leite, mas encontrou BHBA plasmático mais alto numericamente nas vacas de maior ECC. BHBA foi mais alto para o grupo $\mathrm{I}(\mathrm{P}<0,0084)$, devido à perda de peso ter sido mais intensa nas vacas desse grupo.

As médias de cada semana são apresentadas na Tabela 3 .

Quando analisada semanalmente, a glicose foi menor no grupo I durante a primeira e segunda semanas ( $\mathrm{P}<0$ e $\mathrm{P}<0,008$ respectivamente), e com tendência a ser mais baixa nas demais. Concordando com este resultado, este grupo também apresentou BHBA maior que o grupo II durante as semanas $1(\mathrm{P}<0,009), 2(\mathrm{P}<0)$ e $3(\mathrm{P}<0,004)$, sendo que o pico ocorreu na primeira e segunda semanas para ambos os grupos.

A análise do peso e ECC (Tabela 4), revelou que o peso ao parto, a perda de peso e a perda de ECC no pós-parto foram maiores e diferentes ( $\mathrm{P}<0,000, \mathrm{P}<0,010$ e $\mathrm{P}<0$, respectivamente), para o grupo I, sendo que este grupo pesou $99 \mathrm{~kg}$ a mais que o grupo II e perdeu, em média, $25 \mathrm{~kg}$ a mais.

De acordo com SANTOS (1996), as vacas que perdem mais peso no pós-parto têm maior dificuldade em aumentar o consumo de MS, e as vacas que perdem menos peso atingem o "nadir" energético mais cedo e aumentam mais precocemente o consumo voluntário. Considerando-se que a mobilização de reservas corporais pode representar um aumento nos níveis plasmáticos de ácidos graxos não esterificados (AGNE) (PETHICK e DUNSHEA, 1993), estes dados reforçam outros resultados em que vacas mais gordas ao parto

Tabela 2 - Efeito de escore de condição corporal ao parto (Grupos I: ECC $\geq 4,0$ ell: ECC $\leq 3,5$ ), sobre a média total $^{1}$ dos parâmetros metabólicos e de produção

Table 2 - Effect of body condition score at calving (Groups I: $E C C \geq 4.0$ and II: ECC $\leq 3.5$ ), on metabolic and production parameters

\begin{tabular}{|c|c|c|c|}
\hline \multirow[b]{2}{*}{ Item $^{1}$} & \multicolumn{3}{|c|}{$\begin{array}{l}\text { Grupos }^{2} \\
\text { Groups }^{2}\end{array}$} \\
\hline & $\begin{array}{l}\mathrm{I}(\mathrm{n}=23) \\
I(n=23)\end{array}$ & $\begin{array}{l}\mathrm{II}(\mathrm{n}=65) \\
I I(n=65)\end{array}$ & $\begin{array}{c}\mathrm{P}< \\
\text { P value } \\
\end{array}$ \\
\hline $\begin{array}{l}\text { Produção de leite, } \mathrm{kg} / \text { dia } \\
\text { Milk production, } \mathrm{kg} / \text { day }\end{array}$ & 23,70 & 23,10 & 0,704 \\
\hline $\begin{array}{l}\text { Gordura do leite, } \% \\
\text { Milk fat, } \%\end{array}$ & 3,57 & 3,47 & 0,218 \\
\hline $\begin{array}{l}\mathrm{BHBA}^{3}, \mathrm{mg} / \mathrm{dL} \\
B H B A^{3}, m g / d L\end{array}$ & 4,11 & 3,13 & 0,008 \\
\hline $\begin{array}{l}\text { Glicose, } \mathrm{mg} / \mathrm{dL} \\
\text { Glucose, } m g / d L\end{array}$ & 44,68 & 46,73 & 0,101 \\
\hline $\begin{array}{l}\mathrm{AST}^{4}, \mathrm{UI} \\
A S T^{4}, I U^{5}\end{array}$ & 75,93 & 77,90 & 0,650 \\
\hline
\end{tabular}

1 Média das primeiras oito semanas após o parto.

2 Média dos valores de todas vacas do grupo.

3 Beta-hidroxibutirato.

4 Aspartato-aminotransferase.

5 Unidades internacionais.

1 Average for the first eight weeks post-partum

2 Average values for all cows in the group.

3 Beta-hidrxybutirate.

4 Aspartate-aminotransferase.

5 Internationals units. 
Tabela 3 - Efeito de escore de condição corporal ao parto (Grupos I: ECC $\geq 4,0$ e II: ECC $\leq 3,5$ ), sobre a média semanal dos parâmetros metabólicos e de produção

Table 3 - Effect of body condition score at calving (Groups I: ECC $\geq 4.0$ and II: ECC $\leq 3.5$ ) on weekly metabolic parameters and production

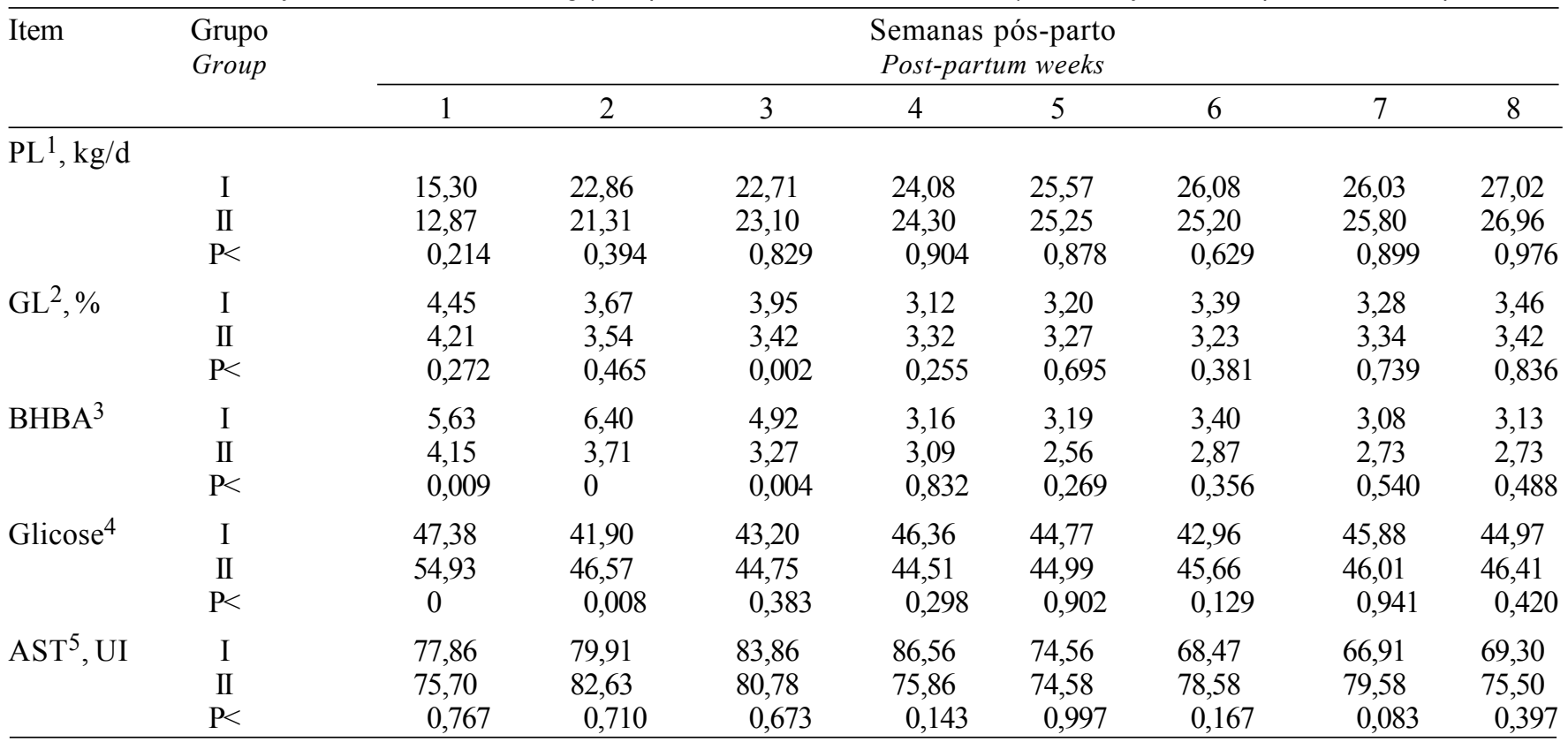

1 Produção de leite, kg/dia (Milk production, $\mathrm{kg} /$ day).

2 Gordura do leite (Milk fat, \%).

${ }^{3}$ Beta-hidroxibutirato (Beta-hydroxybutirate, $\mathrm{mg} / \mathrm{dL}$ ).

${ }^{4}$ Glicose, $\mathrm{mg} / \mathrm{dL}$ (Glucose, $\mathrm{mg} / \mathrm{dL}$ ).

${ }^{5}$ Aspartato-aminotransferase, UI (Aspartate-aminotransferase, IU).

Tabela 4 - Efeito de escore de condição corporal ao parto (Grupos I: ECC $\geq 4,0$ e II: ECC $\leq 3,5$ ), sobre a alteração da condição corporal e peso

Table 4 - Effect of body condition score at calving (Groups I: ECC $\geq 4.0$ and II: ECC $\leq 3.5$ ) on weight and body condition score changes

\begin{tabular}{|c|c|c|c|}
\hline \multirow[b]{2}{*}{ Item $^{1}$} & \multicolumn{3}{|c|}{$\begin{array}{l}\text { Grupos }{ }^{1} \\
\text { Groups }\end{array}$} \\
\hline & $\begin{array}{l}\mathrm{I}(\mathrm{n}=23) \\
I(n=23)\end{array}$ & $\begin{array}{l}\mathrm{II}(\mathrm{n}=65) \\
I I(n=65)\end{array}$ & $\begin{array}{c}\mathrm{P}< \\
\text { Pvalue }\end{array}$ \\
\hline $\begin{array}{l}\text { ECC ao parto } \\
B C S \text { at calving }\end{array}$ & 4,29 & 3,29 & 0 \\
\hline $\begin{array}{l}\text { ECC final }^{2} \\
\text { Final } B C S^{2}\end{array}$ & 3,02 & 2,71 & 0,0003 \\
\hline $\begin{array}{l}\text { Perda de } \mathrm{ECC}^{3} \\
B C S \text { change }\end{array}$ & $-1,27$ & $-0,57$ & 0 \\
\hline $\begin{array}{l}\text { Peso ao parto, } \mathrm{kg} \\
\text { Weight at calving, } \mathrm{kg}\end{array}$ & 663 & 564 & 0 \\
\hline $\begin{array}{l}\text { Peso final } \\
\text { Final weight }^{2}\end{array}$ & 603,39 & 529,38 & 0 \\
\hline $\begin{array}{l}\text { Perda de peso } \\
{ }^{3}, \mathrm{~kg} \\
\text { Weight }_{\text {change }}{ }^{3}, \mathrm{~kg}\end{array}$ & $-59,60$ & $-34,61$ & 0,010 \\
\hline $\begin{array}{l}{ }^{1} \text { Média dos valores de } \\
2 \text { Observação na } 8^{a} \text { se } \\
{ }^{3} \text { Observação da } 8^{a} \text { ser } \\
{ }^{1} \text { Average for all cows. } \\
2 \text { Observation on the } 8^{\text {th }} \text { we }\end{array}$ & $\begin{array}{l}\text { acas do } \\
\text { s-parto } \\
\text { nos a } 1^{\text {a }}\end{array}$ & nana. & \\
\hline
\end{tabular}

Tabela 5 - Efeito de escore de condição corporal ao parto (Grupos I: ECC $\geq 4,0$ e II: ECC $\leq 3,5$ ), sobre a incidência de doenças ${ }^{1}$

Table 5 - Effect of body condition score at calving (Groups I: ECC $\geq 4.0$ and II: $E C C \leq 3.5$ ), on disease occurence ${ }^{1}$

\begin{tabular}{lcccc}
\hline Item & $\begin{array}{c}\text { Grupo I } \\
\text { Group I } \\
(\mathrm{n}=23)\end{array}$ & $\begin{array}{c}\text { Grupo II } \\
\text { Group II } \\
(\mathrm{n}=65)\end{array}$ & $\left(\chi^{2}\right)$ & $\mathrm{P}<$ \\
\hline $\begin{array}{l}\text { Cetose } \\
\text { Ketosis }\end{array}$ & 5 & 6 & 2,930 & 0,119 \\
$\begin{array}{l}\text { Distocia } \\
\text { Dystocia }\end{array}$ & 1 & 11 & 2,280 & 0,130 \\
$\begin{array}{l}\text { RMF } \\
\text { RFM }\end{array}$ & 11 & 24 & 0,840 & 0,359 \\
$\begin{array}{l}\text { Infeção uterina } \\
\begin{array}{l}\text { Uterine infection } \\
\text { Paresia da parturiente }\end{array}\end{array}$ & 1 & 45 & 0,538 & 0,463 \\
$\begin{array}{l}\text { Parturient Paresis } \\
\text { Mastite } \\
\begin{array}{l}\text { Mastitis } \\
\text { Claudicação }\end{array}\end{array}$ & 1 & 0 & 2,860 & 0,091 \\
$\begin{array}{l}\text { Lameness } \\
\text { Cisto ovariano } \\
\text { Ovarian cyst }\end{array}$ & 4 & 3 & 3,790 & 0,052 \\
\hline
\end{tabular}

${ }^{1}$ Número de vacas apresentando sintomas em pelo menos 1 semana do experimento.

2 Retenção de membranas fetais por pelo menos 12 horas após o parto.

1 Number of cows showing symptoms at least in one week of the experiment.

2 Retained fetal membranes for at least 12 hours post-partum. 
mobilizaram mais reservas corporais e tenderam a perder mais peso (REID et al., 1986; PEDRON et al., 1993), havendo uma relação inversa entre consumo de alimento e mobilização de gordura, conforme demonstrado por BERTICS at al. (1992), GRUMMER (1993), STUDER et al. (1993) e VAZQUEZ-ANON et al. (1994). Entretanto, não houve diferença na incidência de cetose (Tabela 5), entre os grupos $(\mathrm{P}<0,119)$. Isto concorda com outros autores que também não puderam associar alta condição corporal ao parto e incidência de doenças (GARNSWORTHY e TOPPS, 1987; BUTLER e SMITH, 1989; GEARHART et al., 1990; RUEGG e MILTON, 1995; SANTOS, 1996).

\section{Conclusões}

O escore de condição corporal ao parto não afetou a produção de leite, teor de gordura do leite, teores plasmáticos de glicose e aspartato-aminotransferase e a incidência de doenças no período pós-parto.

\section{Referências Bibliográficas}

ASSOCIATION OF OFFICIAL ANALYTICAL CHEMISTS. AOAC. 1990. Official Methods of Analysis. 15. ed. Washington, D.C. 1298 p.

BERTICS, S.J., GRUMMER, R.R., CADORNIGA-VALINO, C. et al. 1992. Effect of prepartum dry matter intake on liver triglyceride concentration in early lactation. J. Dairy Sci., 75(7):1914-1922.

BUTLER, W.R., SMITH, R.D. 1989. Interrelationships between energy balance and postpartum reproductive function in dairy cattle. J. Dairy Sci., 72(3):767-783.

CURTIS, C.R., ERB, H.N., SNIFFEN, C.J. et al. 1985. Path analysis of dry period nutrition, postpartum metabolic and reproductive disorders an mastitis in Holstein cows. J. Dairy Sci., 68(9): 2347-2360.

DOMECQ, J.J., SKIDMORE, A.L., LOID, J.W. et al. 1997. Validation of body condition scores with ultrasound measurements of subcutaneous fat of dairy cows. J. Dairy Sci., 78(10):2308-2313.

EDMONSON, A.J., LEAN, I.J., WEAVER, L.D. et al. 1989. A body condition scoring chat for Holstein dairy cows. J. Dairy Sci., 72(1):68-78.

FERGUSON, J.D. 1991. Nutrition and reproduction in dairy cows. Dairy nutrition management. In: Veterinary Clinics of North America. Food Animal Practice, 7(2):483-507.

FERGUSON, J.D., BYERS, D., FERRY, J. et al. 1994. Round table discussion: body condition of lactating cows. Agric. Practice, 15(4):17-21.

GARNSWORTHY,P.C., TOPPS, J.H. 1987. The effect of body condition of dairy cows at calving on their food intake and performance when given complete diets. Anim. Prod., 35(1):113-119.
GEARHART, M.A., CURTIS, C.R., ERB, H.N. et al. 1990. Relationship of change in condition score to cow health in Holsteins. J. Dairy Sci., 73(11):3132-3140.

GRUMMER, R.R. 1993. Etiology of lipid-related metabolic disorders in periparturient dairy cows. J. Dairy Sci., 76(12):3882-3896.

LATOUR, D. 1994. “Getting Started with PROC MIXED”. SAS Institute Inc, Cary, NC. 121p.

NATIONAL RESEARCH COUNCIL - NRC. 1989. Nutrient requeriments of dairy cattle. 6.ed., Rev. Edition, Washington D.C.: National Academy Press. 157p.

PATTON, R.A., BUCHOLTZ, H.F., SCHMIDT, M.K. et al. 1988. Body condition scoring: a management tool. Dairy Guide, East Lansing: Michigan. 6p.

PEDRON, O., CHELI, F., SENATORE, E. et al. 1993. Effect of body condition score at calving on performance, some blood parameters, and milk fatty acid composition in dairy cows. J. Dairy Sci., 76(9):2528-2535.

PETHICK, D.W., DUNSHEA, F.R. 1993. Fat metabolism and turnover. In: FORBS, J.M., FRANCE, J. (Eds.) Quantitative Aspects of ruminant digestion and betabolism. Cambridge: C.A.B. International. 1.ed. p.291-311.

REID, I.M., ROBERTS, C.J., TREACHER, R.J. et al. 1986. Effect of body condition at calving on tissue mobilization, development of fatty liver and blood chemistry of dairy cows. Anim. Prod., 43(1):7-15.

RUEGG, P., MILTON, R.L. 1995. Body condition score of Holstein cows on Prince Edward Island, Canada: Relationships with milk yield, reproductive performance, and disease. J. Dairy Sci., 78(3):552-564.

SANTOS, J.E.P. Effect of degree of fatness prepartum on lactational performance and follicular development of early lactating dairy cows. Tucson, AZ:1996.107p. Thesis (Master of Animal Science)- University of Arizona, 1996.

STUDER, V.A., GRUMMER, R.R., BERTICS, S.J. 1993. Effect of prepartum propylene glycol administration on periparturient fatty liver in dairy cows. J. Dairy Sci., 76(10):2931-2939.

TIETZ, N.W. 1982. Fundamental of clinical chemistry. 2.ed., Philadelphia: Saunders. 240p.

VAN SOEST, P.J., ROBERTSON, J.B., LEWIS, B.A. 1991. Methods for dietary fiber, neutral detergent fiber, and nonstarch polysaccharides in relation to animal nutrition. J. Dairy Sci., 74(12):3583-3597.

VAZQUEZ-ANON, M., BERTICS, S., LUCK, M . et al. 1994. Peripartum liver triglyceride and plasma metabolites in dairy cows. J. Dairy Sci., 77(6):1521-1528.

WILDMAN, E.E., JONES, G.M., WAGNER, P.E. et al. 1982. A dairy body condition scoring system and its relationship to selected production characteristics. J. Dairy Sci., 65(3):495-501.

WILLIANSON, D.H., MELANBY, J., KREBS, H.A. 1962. Enzymatic determination of D(-)-b-hydroxybutyric acid and acetoacetic acid in blood. Biochemistry J., 82(1):90-96.
Recebido em: 19/09/00 Aceito em: 09/05/01 